\title{
Lipoprotein Ratios: A Potential Biomarker for Clinical Diagnosis of Atherosclerosis in Type 1 Diabetic Patients With Foot Ulceration
}

\author{
Walid Hassene Hamri ${ }^{1}$, Mustapha Diaf ${ }^{1}$ \\ 1. Department of Biology, Djillali Liabes University, Sidi Bel Abbes, DZA
}

Corresponding author: Walid Hassene Hamri, walidhamri94@gmail.com

\section{Abstract \\ Background}

Lipoprotein ratios are indicators of atherosclerosis and related diseases such as cardiovascular diseases (CVDs). Early and accurate diagnosis of atherosclerotic disease in patients with diabetic foot ulceration (DFU) is required urgently and remains fundamental to assess the risk of CVDs. This study aimed to determine whether lipoprotein ratios can predict atherosclerosis in type 1 diabetic patients with DFU.

\section{Methodology}

This was a cross-sectional study including 255 patients with confirmed type 1 diabetes with a male-tofemale ratio of 1.19. Patients admitted to the hospital due to diabetes-related complications were divided into the following groups: patients without DFU $(n=153)$ and patients with DFU $(n=102)$. Clinical, biological, and pathophysiological features of patients were compared.

\section{Results}

Our study reported a distinct predominance of males (54.50\%), with a mean age of $28.64 \pm 10.92$ years and duration of diabetes of $10.40 \pm 9.25$ years. The prevalence of DFU was $40.0 \%$. The receiver operator characteristic curve was applied to define the best cut-off lipid ratios to detect atherosclerosis. Total cholesterol (TC)/high-density lipoprotein (HDL) ratio was a valid marker for atherosclerosis with a sensitivity of $86.3 \%$, specificity of $71.4 \%$, and diagnostic accuracy of $0.836 \%$. The findings showed that the fourth quartiles (odds ratio $[\mathrm{OR}]=83.02[22.18-310.75] ; \mathrm{p}=<10^{-3}$ ) of TC/HDL ratio was significantly higher in patients with DFU. Similarly, the last quartiles (fourth) of low-density lipoprotein (LDL)/HDL and triglyceride (TG)/HDL ratio were higher in DFU group (OR = 33.71 [12.04-94.38], $\mathrm{p}=<10^{-3}$; OR $=9.60$ [4.2721.58 ], $\mathrm{p}=<10^{-3}$; respectively). In the DFU group, conventional lipid profiles and lipid ratios were markedly higher in males compared to females patients $(\mathrm{TG}=1.31 \pm 0.69 \mathrm{~g} / \mathrm{L}$ vs. $1.04 \pm 0.84 \mathrm{~g} / \mathrm{L}, \mathrm{p}=0.04$; respectively; $\mathrm{TC} / \mathrm{HDL}=4.79 \pm 1.04$ vs. $4.22 \pm 0.98, \mathrm{p}=0.03$; respectively; $\mathrm{LDL} / \mathrm{HDL}=2.91 \pm 1.13$ vs. $2.17 \pm 1.28, \mathrm{p}=0.01$; respectively; $\mathrm{TG} / \mathrm{HDL}=3.65 \pm 2.53$ vs. $2.67 \pm 1.94, \mathrm{p}=0.008$; respectively).

Review began 03/06/2021 Review ended 03/19/2021 Published 03/23/2021

\section{() Copyright 2021}

Hamri et al. This is an open access article distributed under the terms of the Creative Commons Attribution License CC-BY 4.0., which permits unrestricted use, distribution, and reproduction in any medium, provided the original author and source are credited.

\section{Conclusions}

Elevated atherogenic indices were significantly associated with the atherosclerotic load in patients with DFU, supporting the use of lipid ratios as a biomarker for the diagnosis of atherosclerosis disease in clinical practice in the future.

Categories: Cardiology, Endocrinology/Diabetes/Metabolism, Epidemiology/Public Health

Keywords: lipoprotein ratios, atherosclerosis, cardiovascular diseases, diabetic foot ulceration, type 1 diabetes

\section{Introduction}

Diabetic foot complications are contributing to long-term morbidity and mortality among the diabetic population leading to repeated hospitalizations and high treatment costs for the patients and community at large [1]. It is estimated that $24.4 \%$ of the entire healthcare expenditure among the diabetic population is related to foot complications [2]. The risk of diabetic foot ulceration (DFU) and amputation among diabetic patients increases by two to four folds with the progression of age and diabetes duration regardless of the type of diabetes [3]. It has also been proven by many longitudinal epidemiological studies that among diabetic patients, the prevalence of foot ulcers is about $15 \%$, and the lifetime incidence of DFU may be up to $25 \%$ [4]. Therefore, the main treatment for DFU is ensuring tissue oxygenation by reducing insufficient tissue nourishment induced by increased atherosclerosis and reduced angiogenesis [5].

A prominent feature of type 1 diabetes (T1D) is atherogenic dyslipidemia, characterized by an increase in triglyceride (TG)-rich lipoproteins, a decrease in plasma levels of high-density lipoprotein (HDL) cholesterol (TC), and an increase in the levels of low-density lipoprotein (LDL) particles [6]. Oxidation of LDL is a key 
process in the early progression of atherosclerotic diseases and T1D complications [7]. The use of plasma and serum biomarkers for the prediction and diagnosis of atherosclerosis can be a noninvasive and widely available resource for clinical management. The atherogenic indices of plasma known as Castelli's risk index-I (TC/HDL-c), Castelli's risk index-II (LDL-c/HDL-c), and the logarithmic ratio of TG to HDL-c concentration [8] are indicators of atherosclerosis and related diseases such as cardiovascular dysfunction and peripheral artery disease $(\mathrm{PAD})[9-11]$.

Defined as the atherosclerotic occlusive disease of the lower limbs, PAD is associated with an increased risk of lower extremity amputation and serves as a biomarker for atherothrombosis in cardiovascular beds [12]. Additionally, PAD can cause severe and prolonged disability in diabetic patients [12]. Furthermore, these patients are at the greatest risk for cardiovascular disease (CVD) morbidity compared with diabetic patients who do not have DFU [13]. Hence, focused clinical examination of the feet, particularly palpation of foot pulses, remains fundamental to assess the risk of CVD [14]. Nevertheless, palpation of foot pulses, especially in the presence of DFU, can be unreliable in screening for the presence of PAD in people with diabetes [14].

Thus, to test the concept that T1D patients with DFU have an increased prevalence of atherosclerotic load, as shown using clinical, immunological, and biological measurements such as blood lipid ratios, we investigated the association between these measures and DFU in a group of patients with T1D but without previous CVD.

\section{Materials And Methods}

This was a cross-sectional, observational study covering data from January 1, 2013 to December 31, 2019 on patients with T1D who attended the Diabetes-Endocrinology Department of the University Hospital Center in Sidi Bel Abbes, northwestern Algeria. The study sample consisted of 255 T1D patients diagnosed in their pubertal period (according to the World Health Organization's criteria) who were over age 13 years at the time of the study. Patients admitted to the hospital due to diabetes-related complications were divided into the following groups: patients without DFU $(n=153)$ and patients with DFU $(n=102)$. All the files of the patients were reviewed for the following: medical history, other associated diseases, biochemical parameters, and complications of the diabetic disease. All T1D patients aged more than 13 years, who had angiography with an antegrade approach and had plain radiography of the foot, and visited the DiabetesEndocrinology Department at least one time a year with no history of any CVD were enrolled in the analysis. We excluded around 80 patients due to missing valid information on the disease status and missing informed consent.

For all patients, anthropometry parameters including body weight and height, body mass index (BMI), and waist circumference were available in the patient's medical record. Blood pressure was measured using a sphygmomanometer in a supine position followed by a second measurement (after a few minutes) in a standing position. Hypertension was defined by systolic blood pressure (SBP) of $140 \mathrm{mmHg}$ and diastolic blood pressure (DBP) of about $90 \mathrm{mmHg}$ or more. The latest biochemical assessment including fasting blood glucose, glycated hemoglobin (HbA1c), hemoglobin $(\mathrm{Hb})$, high-sensitivity C-reactive protein (hs-CRP), urea, serum creatinine, urinary albumin excretion rate (UAER), and lipid parameters, namely, total cholesterol (TC), HDL, LDL, and TG, were measured in the hospital during the period of hospitalization by MINDRAY BS-230 using a commercial kit. All data were taken from patients' medical records. The ulcers in patients with diabetic foot were graded from 1 to 5 according to the presence of infection and/or gangrene using the Wagner Classification [15] (grade 1: presence of superficial ulcer limited to the epidermis; grade 2: infection reaching the dermis, muscle, tendon, and ligaments but no signs of osteomyelitis; grade 3: presence of deep soft tissue infection and osteomyelitis; grade 4: gangrene localized to the distal foot; grade 5: extensive gangrene). Examination findings and culture and radiology results were utilized to classify the foot ulcers. Moreover, lipid ratios as indicators of atherogenic risk were calculated, namely, TC/HDL, LDL/HDL, and TG/HDL.

Due to the sample size, all data were compiled and analyzed using SPSS version 22 (IBM Corporation, Chicago, IL, USA). The descriptive statistics of mean \pm standard deviation with its respective $95 \%$ confidence intervals (95\% CI) were estimated for quantitative variables, whereas qualitative variables were expressed as percentages (\%) and relative frequencies. Continuous variables underlying normal distribution were compared between groups using Student's t-test, while categorical variables were compared between groups with the Chi-square test. Odds ratios (OR) and 95\% CIs for lipid profiles were determined using multivariate logistic regression analysis after adjustment across quartiles of lipid ratios to investigate the association between DFU and atherosclerosis. The receiver operator characteristic (ROC) curve was used to determine the best cutoff value and validity of lipid ratios, and the area under the ROC curve (AUC), sensitivity, specificity, positive predictive value (PPV), and negative predictive value (NPV) were calculated. Statistically significant differences were maintained when the p-value was less than or equal to 0.05 .

\section{Results}

The basic characteristics of the studied patients are shown in Table 1 . A total of 255 T1D patients $(54.50 \%$ males and $45.50 \%$ females) were enrolled during the period of the study. For the purpose of the analysis, patients were classified into two groups according to the presence of DFU. Of the 255 diabetic patients, 102 


\section{Cureus}

(40.00\%) patients had DFU. For all patients, the mean age was $28.64 \pm 10.92$ years (range $=13-70$ years), and the mean diabetes duration was $10.40 \pm 9.25$ years. Meanwhile, the mean age of patients with DFU was significantly higher than those without DFU (33.98 \pm 11.98 years vs. $25.07 \pm 8.48$ years, $\mathrm{p}=\left\langle 10^{-3}\right.$, respectively). Similarly, the mean duration of diabetes was significantly higher among those who developed DFU compared to those who did not ( $13.55 \pm 10.32$ years vs. $8.30 \pm 7.81$ years, $\mathrm{p}=<10^{-3}$, respectively) (Table 2). The most affected age group with DFU was the 30-39 years with a rate of $30.40 \%$, followed by the $40-49$ years group with a rate of $26.50 \%$. However, the least affected age group was the $13-19$ years. Notably, history of smoking showed a slight association with the development of DFU $(23.50 \%, p=0.08)$ whereas there were significant differences across the different weight categories $\left(\mathrm{p}=<10^{-3}\right)$ (Table 1$)$. 


\section{Cureus}

\begin{tabular}{|c|c|c|c|c|}
\hline \multirow{2}{*}{ Variables } & All patients $(n=255)$ & Without DFU (n=153) & With DFU (n=102) & \multirow{2}{*}{ P-Value } \\
\hline & Number (\%) & Number (\%) & Number (\%) & \\
\hline \multicolumn{5}{|l|}{ Gender } \\
\hline Male & 139 (54.50\%) & $75(49.00 \%)$ & $64(62.70 \%)$ & \multirow{2}{*}{0.03} \\
\hline Female & $116(45.50 \%)$ & $78(51.00 \%)$ & $38(37.30 \%)$ & \\
\hline \multicolumn{5}{|l|}{ Age groups } \\
\hline 13-19 years & $49(19.20 \%)$ & $48(31.40 \%)$ & $1(1.00 \%)$ & \multirow{6}{*}{$<10^{-3}$} \\
\hline $20-29$ years & $79(31.00 \%)$ & $67(43.80 \%)$ & $12(11.80 \%)$ & \\
\hline 30-39 years & $58(22.70 \%)$ & $27(17.60 \%)$ & $31(30.40 \%)$ & \\
\hline 40-49 years & $36(14.10 \%)$ & $9(5.90 \%)$ & $27(26.50 \%)$ & \\
\hline $50-59$ years & $30(11.80 \%)$ & $2(1.30 \%)$ & $28(27.40 \%)$ & \\
\hline More than 60 years & $3(1.20 \%)$ & $0(0.00 \%)$ & $3(2.90 \%)$ & \\
\hline \multicolumn{5}{|l|}{ Smoking history } \\
\hline Male & $47(18.40 \%)$ & $23(15.00 \%)$ & $24(23.50 \%)$ & 0.08 \\
\hline \multicolumn{5}{|l|}{ Prevalence of weight categories } \\
\hline Underweight, BMI $<18.5 \mathrm{~kg} / \mathrm{m}^{2}$ & $16(6.30 \%)$ & $13(8.50 \%)$ & $3(2.90 \%)$ & \multirow{4}{*}{$<10^{-3}$} \\
\hline Normal weight, BMI $=18.5-25.0 \mathrm{~kg} / \mathrm{m}^{2}$ & $144(56.50 \%)$ & $83(54.30 \%)$ & $61(59.80 \%)$ & \\
\hline Overweight, BMI = 25.0-29.9 kg/ $/ \mathrm{m}^{2}$ & $55(21.60 \%)$ & $38(24.80 \%)$ & $17(16.70 \%)$ & \\
\hline Obesity, $\mathrm{BMI}=30 \mathrm{~kg} / \mathrm{m}^{2}$ & $40(15.60 \%)$ & $19(12.40 \%)$ & $21(20.60 \%)$ & \\
\hline \multicolumn{5}{|l|}{ Other associated complications } \\
\hline Low visual acuity & 66 (25.90\%) & $29(19.00 \%)$ & $37(36.30 \%)$ & 0.002 \\
\hline Diabetic nephropathy & $22(8.60 \%)$ & $6(3.90 \%)$ & $16(15.70 \%)$ & 0.001 \\
\hline tension & $27(10.60 \%)$ & $6(3.90 \%)$ & $21(20.60 \%)$ & $<10^{-3}$ \\
\hline Hypothyroidism & $19(7.50 \%)$ & $14(9.20 \%)$ & $5(4.90 \%)$ & 0.20 \\
\hline perthyroidism & $6(2.40 \%)$ & $2(1.30 \%)$ & $4(3.90 \%)$ & 0.17 \\
\hline Anemia & $73(28.60 \%)$ & $38(24.80 \%)$ & $35(34.30 \%)$ & 0.10 \\
\hline den & ()) & $\%$ & $5(4.90 \%)$ & 0.02 \\
\hline Diabetic retinopathy & $46(18.00 \%)$ & $18(11.80 \%)$ & $28(27.50 \%)$ & 0.002 \\
\hline Ketosis on diabetes & $93(36.50 \%)$ & $43(28.10 \%)$ & $50(49.00 \%)$ & $<10^{-3}$ \\
\hline \multicolumn{5}{|l|}{ Symptoms and signs } \\
\hline Weight loss & $142(55.70 \%)$ & $88(57.50 \%)$ & $54(52.90 \%)$ & 0.34 \\
\hline lyuria-polyddipsia & $238(90.30 \%)$ & $142(92.80 \%)$ & $96(94.10 \%)$ & 0.70 \\
\hline nia & $107(42.00 \%)$ & $66(43.10 \%)$ & $41(40.20 \%)$ & 0.50 \\
\hline Overeating & $121(47.50 \%)$ & .70 & $48(47.00 \%)$ & 0.90 \\
\hline
\end{tabular}

TABLE 1: Basic characteristics of the study participants.

DFU: diabetic foot ulceration; BMI: body mass index

Percentages were compared with Chi-square test, $p \leq 0.05$ was considered as significant 


\section{Cureus}

In the current study, the most common complications in both groups were ketosis on diabetes $(36.50 \%, \mathrm{p}=$ $\left.<10^{-3}\right)$, followed by anemia $(28.60 \%, \mathrm{p}=0.10)$. In contrast to patients without DFU, low visual acuity, diabetic retinopathy, hypertension, diabetic nephropathy, and dyslipidemia were significantly higher in those with DFU $\left(36.30 \%, p=<0.002 ; 27.50 \%, p=<0.002 ; 20.60 \%, p=<10^{-3} ; 15.70 \%, p=0.001 ;\right.$ and $4.90 \%, p=0.02$, respectively) (Table 1 ).

The clinic characteristics for both groups are summarized in Table 2. Concerning the anthropometric measurement on admission, there were higher significant differences in body weight between both groups $(57.87 \pm 12.63 \mathrm{~kg}$ and $62.11 \pm 11.39 \mathrm{~kg}$ for patients without DFU and with DFU, $\mathrm{p}=0.007$, respectively) and BMI $(\mathrm{p}=<0.01)$, while there were no significant differences in waist circumference and body height $(\mathrm{p}=$

$<0.31$ and $\mathrm{p}=<0.15$, respectively). In addition, significantly higher SBP $\left(\mathrm{p}=<10^{-3}\right)$ and $\mathrm{DBP}\left(\mathrm{p}=<10^{-3}\right)$ was found in patients with DFU compared to those without DFU (Table 2).

Interestingly, similar to fasting plasma glucose concentrations, there was a significant difference in HbA1c levels (patients without DFU: $9.32 \pm 2.35 \%$; patients with DFU: $11.38 \pm 2.08 \%, \mathrm{p}=<10^{-3}$ ) between both groups on admission. No significant difference was found between the patients with and without DFU in terms of $\mathrm{Hb}(\mathrm{p}=0.37)$. On the other hand, hs-CRP was markedly higher in patients with DFU than those without DFU $\left(12.33 \pm 5.24 \mathrm{mg} / \mathrm{L}\right.$ vs. $2.30 \pm 1.27 \mathrm{mg} / \mathrm{L}, \mathrm{p}=<10^{-3}$, respectively). With regard to lipid levels, as shown in Table 2, TC, HDL-c, LDL-c, and TG levels differed significantly among the two groups $\left(\mathrm{p}=<10^{-3}, \mathrm{p}\right.$ $=<0.01, \mathrm{p}=<10^{-3}$, and $\mathrm{p}=<10^{-3}$, respectively). Further, it was found that lipid ratios (TC/HDL-c, LDL/HDL-c, and TG/HDL-c) were significantly greater in the group with DFU $\left(\mathrm{p}=<10^{-3}, \mathrm{p}=<10^{-3}\right.$, and $\mathrm{p}=<10^{-3}$, respectively) (Table 2 ). 


\begin{tabular}{|c|c|c|c|c|c|c|c|}
\hline \multirow{2}{*}{ Variables } & \multicolumn{2}{|c|}{ All patients $(n=255)$} & \multicolumn{2}{|c|}{ Without DFU (n=153) } & \multicolumn{2}{|c|}{ With DFU (n=102) } & \multirow{2}{*}{ P-Valu } \\
\hline & Mean $\pm S D$ & $95 \% \mathrm{Cl}$ & MeantSD & $95 \% \mathrm{Cl}$ & MeantSD & $95 \% \mathrm{Cl}$ & \\
\hline Mean age (years) & $28.64 \pm 10.92$ & 27.29-29.98 & $25.07 \pm 8.48$ & 23.72-26.43 & $33.98 \pm 11.98$ & 31.63-36.34 & $<0^{-3}$ \\
\hline Diabetes duration (years) & $10.40 \pm 9.25$ & $9.26-11.54$ & $8.30 \pm 7.81$ & $7.05-9.55$ & $13.55 \pm 10.32$ & $11.52-15.58$ & $<0^{-3}$ \\
\hline Age at first diagnosis (years) & $18.32 \pm 8.21$ & 17.30-19.33 & $16.88 \pm 8.05$ & 15.59-18.16 & $20.48 \pm 8.02$ & 18.90-22.06 & 0.001 \\
\hline Body height $(m)$ & $1.68 \pm 0.09$ & 1.67-1.69 & $1.67 \pm 0.09$ & $1.66-1.69$ & $1.69 \pm 0.08$ & $1.67-1.71$ & 0.15 \\
\hline Body weight (kg) & $59.57 \pm 12.31$ & $58.05-61.09$ & $57.87 \pm 12.63$ & 55.86-59.89 & $62.11 \pm 11.39$ & 59.87-64.35 & 0.007 \\
\hline BMI (kg/m²) & $20.97 \pm 3.92$ & 20.99-21.46 & $20.50 \pm 3.96$ & 19.87-21.14 & $21.68 \pm 3.79$ & 20.93-22.42 & 0.01 \\
\hline Waist circumference $(\mathrm{cm})$ & $81.62 \pm 11.03$ & 79.23-84.01 & $80.45 \pm 11.74$ & 76.88-84.03 & $82.90 \pm 10.18$ & 79.64-86.16 & 0.31 \\
\hline $\mathrm{SBP}(\mathrm{mmHg})$ & $113.3 \pm 12.8$ & $111.8-114.9$ & $110.7 \pm 11.3$ & $108.8-112.5$ & $117.4 \pm 13.8$ & $114.6-120.1$ & $<10^{-3}$ \\
\hline $\mathrm{DBP}(\mathrm{mmHg})$ & $66.9 \pm 8.9$ & $65.8-68.0$ & $65.2 \pm 8.0$ & $63.9-66.5$ & $69.4 \pm 9.7$ & $67.5-71.3$ & $<0^{-3}$ \\
\hline Fasting plasma glucose (g/L) & $2.52 \pm 0.96$ & $2.40-2.64$ & $2.11 \pm 0.57$ & $2.01-2.20$ & $3.13 \pm 1.10$ & $2.92-3.35$ & $<0^{-3}$ \\
\hline HbA1c (\%) & $10.55 \pm 2.34$ & 10.25-10.84 & $9.32 \pm 2.35$ & $9.11-10.39$ & $11.38 \pm 2.08$ & $10.96-11.80$ & $<10^{-3}$ \\
\hline $\mathrm{Hb}(\mathrm{g} / \mathrm{L})$ & $12.59 \pm 2.03$ & $12.30-12.87$ & $12.69 \pm 1.82$ & $12.36-13.02$ & $12.43 \pm 2.32$ & 11.91-12.95 & 0.37 \\
\hline hs-CRP (mg/L) & $4.99 \pm 5.32$ & $3.70-6.29$ & $2.30 \pm 1.27$ & 1.94-2.67 & $12.33 \pm 5.24$ & $9.72-14.94$ & $<10^{-3}$ \\
\hline TC $(g / L)$ & $1.55 \pm 0.40$ & $1.50-1.60$ & $1.35 \pm 0.20$ & $1.32-1.39$ & $1.85 \pm 0.45$ & $1.76-1.94$ & $<10^{-3}$ \\
\hline HDL-C $(g / L)$ & $0.45 \pm 0.10$ & 0.44-0.46 & $0.46 \pm 0.08$ & $0.45-0.48$ & $0.43 \pm 0.13$ & $0.40-0.46$ & 0.01 \\
\hline LDL-c (g/L) & $0.91 \pm 0.31$ & $0.87-0.95$ & $0.78 \pm 0.18$ & $0.75-0.81$ & $1.12 \pm 0.36$ & 1.04-1.19 & $<10^{-3}$ \\
\hline TG $(g / L)$ & $1.00 \pm 0.64$ & $0.92-1.08$ & $0.80 \pm 0.42$ & $0.73-0.87$ & $1.29 \pm 0.79$ & 1.13-1.44 & $<10^{-3}$ \\
\hline TC/HDL-c & $3.61 \pm 1.29$ & $3.45-3.77$ & $2.96 \pm 0.58$ & $2.87-3.06$ & $4.58 \pm 1.46$ & $4.29-4.87$ & $<10^{-3}$ \\
\hline LDLHDL-C & $2.16 \pm 1.00$ & $2.04-2.29$ & $1.72 \pm 0.51$ & 1.64-1.81 & $2.82 \pm 1.20$ & $2.58-3.05$ & $<10^{-3}$ \\
\hline$T G / H D L-C$ & $2.41 \pm 1.84$ & $2.18-2.63$ & $1.77 \pm 0.98$ & 1.61-1.93 & $3.36 \pm 2.35$ & $2.90-3.32$ & $<10^{-3}$ \\
\hline Creatinine $(\mathrm{mg} / \mathrm{L})$ & $12.38 \pm 12.14$ & 10.12-14.63 & $9.70 \pm 8.07$ & $7.73-11.66$ & $16.20 \pm 15.60$ & $11.61-20.78$ & 0.004 \\
\hline Urea (g/L) & $0.40 \pm 0.33$ & $0.33-0.46$ & $0.32 \pm 0.25$ & $0.26-0.38$ & $0.50 \pm 0.39$ & $0.39-0.62$ & 0.003 \\
\hline Microalluminuria (mg/24h) & $48.37 \pm 73.44$ & 7-6 & $12.77=$ & 10.27-15. & 96 & 71.23-12 & $<10^{-3}$ \\
\hline
\end{tabular}

\section{TABLE 2: Comparison of clinical characteristics between patients with and without DFU.}

DFU: diabetic foot ulceration; SD: standard deviation; Cl: confidence interval; HbA1c: glycosylated hemoglobin; BMI: body mass index; SBP: systolic blood pressure; DBP: diastolic blood pressure; Hb: Hemoglobin; hs-CRP: high-sensitivity C-reactive protein; TC: total cholesterol; HDL-c: high-density lipoprotein cholesterol; LDL-c: low-density lipoprotein cholesterol; TG: triglycerides

Means were compared with independent sample Student's t-test; a $p \leq 0.05$ was considered as significant

With respect to renal function, patients with DFU had increased plasma levels of creatinine and urea and microalbuminuria $(16.20 \pm 15.60 \mathrm{mg} / \mathrm{L}, \mathrm{p}=0.004 ; 0.50 \pm 0.39 \mathrm{~g} / \mathrm{L}, \mathrm{p}=0.003$; and $96.50 \pm 92.59 \mathrm{mg} / 24 \mathrm{~h}, \mathrm{p}=$ $<10^{-3}$, respectively) (Table 2 ).

For the entire study population, the multivariate regression between lipid ratios quartiles, in terms of their strong association with atherosclerosis, plotted that the fourth quartiles (OR $=83.02$ [22.18-310.75], $\mathrm{p}=\left\langle 10^{-}\right.$ ${ }^{3}$ ) of TC/HDL ratio was significantly higher in patients with DFU, as described in Table 3. Similarly, the last quartiles (fourth) of LDL/HDL and TG/HDL ratios were higher in DFU group (OR = 33.71 [12.04-94.38], $\mathrm{p}=$ $<10^{-3} ; \mathrm{OR}=9.60[4.27-21.58], \mathrm{p}=<10^{-3}$, respectively) (Table 3 ). 


\section{Cureus}

\begin{tabular}{|c|c|c|c|c|}
\hline \multirow{2}{*}{ Variables } & Without DFU (n=153) & With DFU (n=102) & \multirow{2}{*}{ OR (95\% Cl) } & \multirow{2}{*}{ P-Value } \\
\hline & Number (\%) & Number (\%) & & \\
\hline \multicolumn{5}{|l|}{ TC/HDL ratio } \\
\hline First quartile (1.42-2.72) & $49(32.0 \%)$ & $12(11.8 \%)$ & Reference & -- \\
\hline Second quartile (2.73-3.27) & $55(35.9 \%)$ & $10(9.8 \%)$ & $0.74[0.29-1.86]$ & 0.52 \\
\hline Third quartile (3.28-4.22) & $46(30.1 \%)$ & $19(18.6 \%)$ & $1.68[0.73-3.85]$ & 0.21 \\
\hline Fourth quartile (4.23-8.21) & $3(2.0 \%)$ & $61(59.8 \%)$ & 83.02 [22.18-310.75] & $<10^{-3}$ \\
\hline \multicolumn{5}{|l|}{ LDL/HDL ratio } \\
\hline First quartile (0.33-1.45) & $48(31.4 \%)$ & $14(13.7 \%)$ & Reference & $\cdots$ \\
\hline Second quartile (1.46-1.91) & $53(34.6 \%)$ & $13(12.7 \%)$ & $0.84[0.35-1.96]$ & 0.69 \\
\hline Third quartile (1.92-2.56) & $46(30.1 \%)$ & $16(15.7 \%)$ & $1.19[0.52-2.71]$ & 0.67 \\
\hline Fourth quartile (2.57-5.97) & $6(3.9 \%)$ & $59(57.9 \%)$ & 33.71 [12.04-94.38] & $<10^{-3}$ \\
\hline \multicolumn{5}{|l|}{$\mathrm{TG} / \mathrm{HDL}$ ratio } \\
\hline First quartile (0.31-1.23) & $48(31.4 \%)$ & $16(15.7 \%)$ & Reference & $\cdots$ \\
\hline Second quartile (1.24-1.88) & $52(34.0 \%)$ & $12(11.8 \%)$ & $0.69[0.29-1.61]$ & 0.39 \\
\hline Third quartile (1.89-2.79) & $38(24.8 \%)$ & $26(25.4 \%)$ & $2.05[0.96-4.36]$ & 0.06 \\
\hline Fourth quartile (2.80-14.95) & $15(9.8 \%)$ & $48(47.1 \%)$ & $9.60[4.27-21.58]$ & $<10^{-3}$ \\
\hline
\end{tabular}

\section{TABLE 3: Crude OR of blood lipid ratios quartiles associated with DFU.}

DFU: diabetic foot ulceration; Cl, confidence interval; OR, odds ratio; TC: total cholesterol; LDL: low-density lipoprotein cholesterol; HDL: highdensity lipoprotein cholesterol; TG: triglycerides

Multivariate logistic regression significant at $p \leq 0.05$

ROC curve was applied to define the best cut-off lipid ratios to detect atherosclerosis. TC/HDL ratio was a valid marker for atherosclerosis. The optimum cut-off value was $\geqslant 4.0$, with a sensitivity of $86.3 \%$, specificity 71.4\%, PPV 73.2\%, and NPV 52.6\% with a diagnostic accuracy of 0.836 . However, LDL/HDL ratio was a moderately valid marker for atherosclerosis. The optimum cut-off value was $\geqslant 2.5$, with a sensitivity of $69.8 \%$, specificity $66.2 \%$, PPV $60.8 \%$, and NPV $47.1 \%$ with a diagnostic accuracy of 0.772 . As for TG/HDL ratio, the optimum cut-off value was $\geqslant 3.0$, with a sensitivity of $64.1 \%$, specificity $61.2 \%$, PPV $57.3 \%$, and NPV $50.4 \%$ with a diagnostic accuracy of 0.740 (Figure 1 ). 


\section{Cureus}

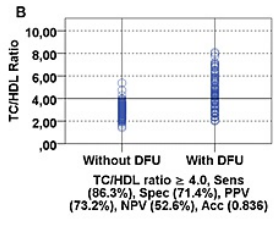

A
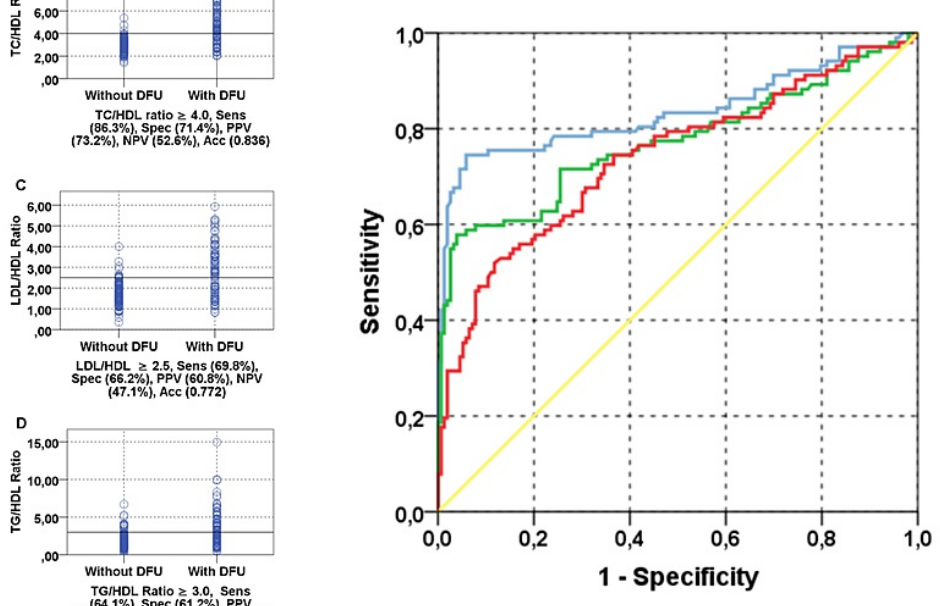

Lipid ratios

- TC/HDL

一 LDLIHDL

-TGIHDL

Reference line

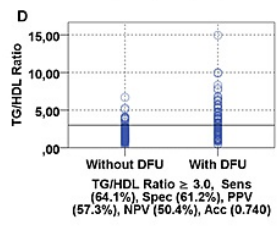

1 - Specificity

\section{FIGURE 1: ROC curve to define the best cut-off lipid ratios to detect} atherosclerosis.

DFU: diabetic foot ulceration; Sens: sensitivity; Spec: specificity; PPV: positive predictive value; NPV: negative predictive value; Acc: accuracy; TC: total cholesterol; LDL: low-density lipoprotein cholesterol; HDL: high-density lipoprotein cholesterol; TG: triglycerides; ROC: receiver operating characteristics

As shown in Figure $2 A-2 C$, in males and females, when comparing all lipid ratios (TC/HDL, LDL/HDL, TG/HDL) between the two groups, higher levels were found in males compared to females with DFU. As for conventional lipid parameters (TC and LDL), higher values were found in females compared to males with DFU (Figures 2D, 2E). 
A

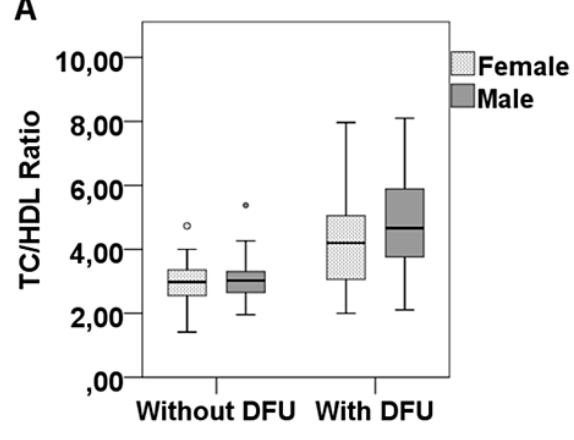

B

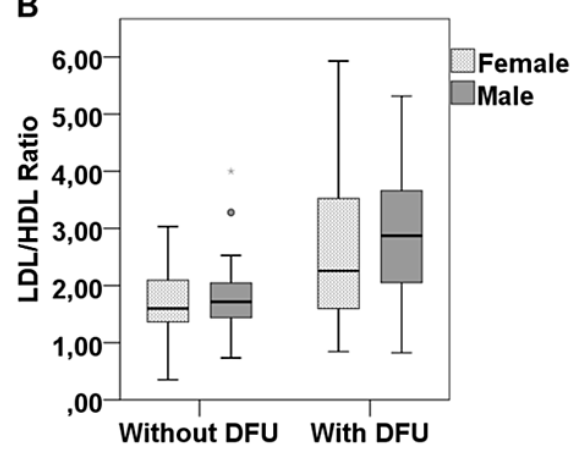

C

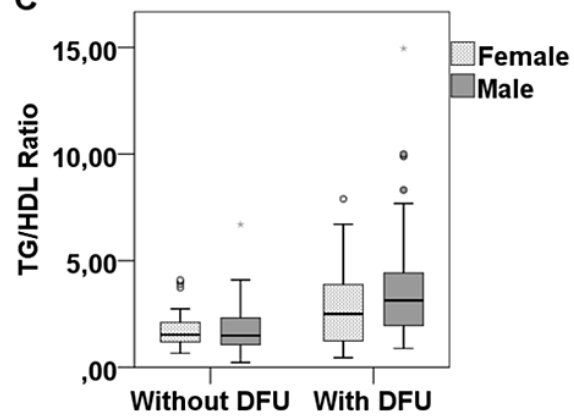

D

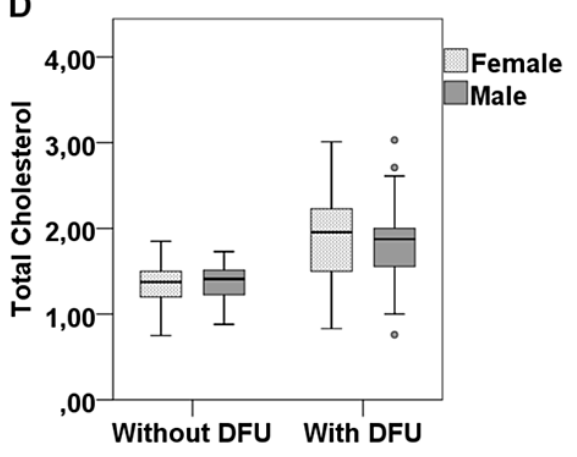

E

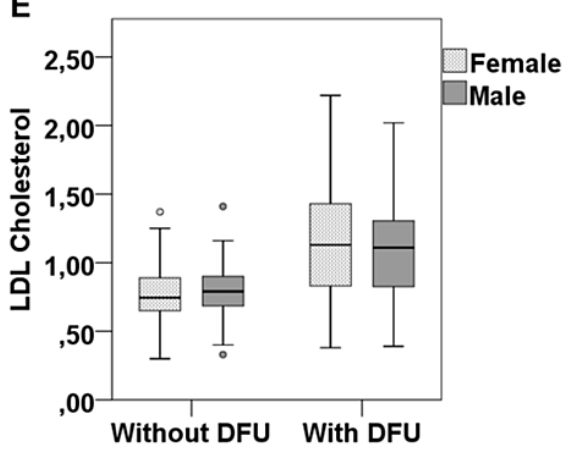

$\mathbf{F}$

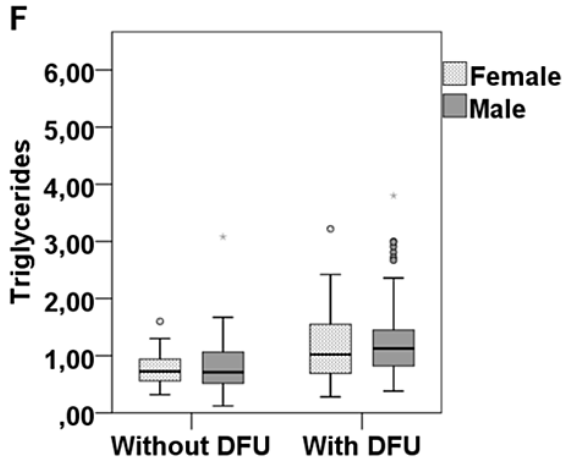

FIGURE 2: Comparison of lipid ratios levels between patients with and without DFU according to their gender.

DFU: diabetic foot ulceration; TC: total cholesterol; LDL: low-density lipoprotein cholesterol; HDL: highdensity lipoprotein cholesterol; TG: triglycerides

\section{Discussion}

In this study, we evaluated the potential role of clinical, immunological, and biological features in patients with prolonged duration of T1D with and without DFU to identify the variables linked to the presence of DFU in this population. Interestingly, apart from other well-established CV risk factors, our findings support the theory that macrovascular diseases such as DFU may contribute to the development of CVD in patients with T1D. Thus, we investigated the association between DFU and the occurrence of atherosclerotic disease (via lipid ratios) by comparing two groups of patients according to the presence of DFU (patients without DFU vs. patients with DFU). Our first observation was that the distribution of patients by gender was unequal by demonstrating a distinct predominance of males over females (54.50-45.50\%), with a male to female ratio of 1.19. Among patients with DFU (40.0\%), the prevalence of foot ulceration was significantly higher in males than in females with T1D. A large retrospective cohort study conducted by Al-Rubeaan et al. [16] showed that 2,071 patients with diabetes had DFU; of those, 1,420 (68.57\%) were men; whereas, in the study by Bruun et al., a follow-up study group, 38 (2.8\%) patients had DFU at diabetes diagnosis. Of the 38 patients, 27 (71.0\%) were men. After a six-year follow-up, 28 (2.9\%) had DFU, and of those, 18 (64.3\%) were men [17]. 
Our study displayed significant impacts of age and diabetes duration on the prevalence of DFU. The correlation of age and diabetes duration with DFU risk is similar to studies conducted by several authors, for example, Nehring et al. [18]. According to the study by Al-Maskari, the longer the duration of diabetes, the greater is the risk of developing DFU [19]. In a follow-up study by Al-Delaimy et al., the relative risk (95\% CI) for DFU compared with patients without diabetes was (1.39 [0.82-2.36]) for one to five years of diabetes, (3.63 [2.23-5.88]) for six to 10 years of diabetes, $(2.55$ [1.50-4.32]) for 11 to 25 years of diabetes, and (4.53 [2.39-8.58]) for more than 25 years of diabetes [20]. The present study also shows that cigarette smoking increased the probability of DFU in the entire study population. A similar conclusion was proffered by AlRubeaan et al. with reference to patients with type 2 diabetes [16]. In the present study, body corpulence increased the risk of DFU. Nehring et al. investigated the effect of BMI on the progression of DFU and concluded that obese diabetic patients are prone to developing diabetic foot complications [18]. Hurley et al. described that the risk of the diabetic foot doubles when a patient's weight is higher than the optimal body weight [21]. Diabetic nephropathy and retinopathy increased the prevalence of DFU in the current study, which could be explained by microangiopathic changes [22], which is also the same observation reported in other studies $[16,17,19]$.

In the current study, hypertension was detected in 27 cases, and 21 (20.60\%) cases with DFU were hypertensive. Our findings are in line with the previous studies which prove that the prevalence of DFU increases in hypertensive patients $[12,19,23]$. An important observation of our study was the association of an increased prevalence of DFU with the presence of ketosis in patients with T1D. Our findings echo the observations made by McIntyre who suggested that the avoidance of ketoacidosis may be the key to the prevention of diabetic foot complications [24]. We also observed an incremental increase in HbA1c values in patients with DFU, suggesting a significant relationship between the diabetic foot conditions and the degree of glycemic control. Previous studies reported that poor glycemic control was associated with a two-fold increase in the risk of foot lesions among diabetic patients $[25,26]$.

Our study has shown strong and consistent associations between the amount of hs-CRP and DFU. Statistically significant correlations were shown in a case-control study between DFU and elevated concentrations of hs-CRP [27]. In addition to reduced glomerular filtration rate (as used to define diabetic nephropathy), we further observed a sustained increase in UAER ( $\geqslant 30 \mathrm{mg} / 24 \mathrm{~h}$ ) in the DFU group, which may allow these results to be more reliable. Al-Maskari et al. confirmed that the presence of microalbuminuria is a significant risk factor for foot complications [19]. This was in accordance with a previous follow-up study in newly diagnosed diabetic patients observed for 19 years [17]. Additionally, consistent with several previous studies $[6,27,28]$, we also found that there were higher TG concentrations and lower HDL-c concentrations in the DFU group compared with the group without DFU. These observations suggest that lipid ratios can be utilized to evaluate the occurrence of atherosclerosis and the severity of the disease, supporting the employment of this index as an ideal biomarker for diagnosis in clinical practice [28].

Atherosclerosis is known to occur in the proximal arteries of the diabetic limb and presents as iliac, femoral, and popliteal diseases. The risk factor for this proximal site disease is dyslipidemia [29]. Therefore, as a new comprehensive indicator of blood lipid levels, lipid ratios have received increasing attention and can be particularly useful in predicting the risk of CVD [8]. Furthermore, lipid ratios are more reliable than classic lipid parameters in predicting arterial stiffness and contribute significantly to the estimation of CVD risk and arterial stiffness, especially when the absolute values of lipid profile seem normal or not markedly deranged [30].

Despite strong evidence for the utility of lipid ratios in the diagnosis of atherosclerosis, several limitations exist that warrant further study. First, the design of this cross-sectional study was observational and examined a relatively small number of patients because of constrictive inclusion and exclusion criteria. Second, our study highlights only one extremity with diabetic ulcer and gangrene because we restricted it to those with established ulceration where robust exclusion is more important than specificity. Nevertheless, we believe that our study is important from a public health and clinical perspective. To the best of our knowledge, it is the first study that assessed DFU complications in a moderately large sample of T1D in our population. Another strength of this research is the area-specific analysis of DFU risk among the study patients. This study will help address the regional disparities in diabetes foot care in Algeria and in remodeling the chain of diabetes centers where appropriate.

\section{Conclusions}

In conclusion, this study showed that the atherosclerotic load in patients with DFU is significantly higher than in patients without DFU. Likewise, we found that lipid ratios were significantly associated with DFU and can be used as a biomarker for the diagnosis of atherosclerosis disease in clinical practice in the future. Further, based on the findings of this study, we recommend that specialized diabetes care centers should concentrate more on the contributing factors of DFU in the management of patients with T1D as these are associated with morbidity and mortality resulting from CVD.

\section{Additional Information}

\section{Disclosures}


Human subjects: Consent was obtained or waived by all participants in this study. Ethics Committee of the University Hospital Center issued approval AAU-3/2019. The ethics committee approved this retrospective study, and due to the nature of the study, ethical approval was obtained from the department in which the study was carried out. Animal subjects: All authors have confirmed that this study did not involve animal subjects or tissue. Conflicts of interest: In compliance with the ICMJE uniform disclosure form, all authors declare the following: Payment/services info: All authors have declared that no financial support was received from any organization for the submitted work. Financial relationships: All authors have declared that they have no financial relationships at present or within the previous three years with any organizations that might have an interest in the submitted work. Other relationships: All authors have declared that there are no other relationships or activities that could appear to have influenced the submitted work.

\section{References}

1. Nyamu PN, Otieno CF, Amayo EO, McLigeyo SO: Risk factors and prevalence of diabetic foot ulcers at Kenyatta National Hospital, Nairobi. Afr Med J. 2003, 80:36-43. 10.4314/eamj.v80i1.8664

2. Sargen MR, Hoffstad O, Margolis DJ: Geographic variation in Medicare spending and mortality for diabetic patients with foot ulcers and amputations. J Diabetes Complications. 2013, 27:128-133. 10.1016/j.jdiacomp.2012.09.003

3. Katsilambros N, Tentolouris N, Tsapogas P, Dounis E: Who is the patient at risk for foot ulceration? . Atlas of Diabetic Foot. John Wiley \& Sons, Chichester, UK; 2003. 1-21. 10.1002/047086138X.ch1

4. Yazdanpanah L, Shahbazian H, Nazari I, et al.: Incidence and risk factors of diabetic foot ulcer: a population-based diabetic foot cohort (ADFC Study)-two-year follow-up study. Int J Endocrinol. 2018, 2018:7631659. 10.1155/2018/7631659

5. Zimny S, Schatz H, Pfohl M: The role of limited joint mobility in diabetic patients with an at-risk foot . Diabetes Care. 2004, 27:942-946. 10.2337/diacare.27.4.942

6. Chapman MJ: The effects of fibrates on lipid metabolism and inflammation in type 2 diabetes . Diabetes Vasc Dis Res. 2006, 3:6-9. 10.1177/147916410600300103

7. Renie G, Maingrette F, Li L: Diabetic vasculopathy and the lectin-like oxidized low-density lipoprotein receptor-1 (LOX-1). Curr Diabetes Rev. 2007, 3:103-110. 10.2174/157339907780598225

8. Bhardwaj S, Bhattacharjee J, Bhatnagar MK, Tyagi S: Atherogenic index of plasma, castelli risk index and atherogenic coefficient-new parameters in assessing cardiovascular risk. Int J Pharm Biol Sci. 2013, 3:359364.

9. Kannel WB, Vasan RS, Keyes MJ, Sullivan LM, Robins SJ: Usefulness of the triglyceride-high-density lipoprotein versus the cholesterol-high-density lipoprotein ratio for predicting insulin resistance and cardiometabolic risk (from the Framingham Offspring Cohort). Am J Cardiol. 2008, 101:497-501. 10.1016/j.amjcard.2007.09.109

10. Nwagha UI, Ikekpeazu EJ, Ejezie FE, Neboh EE, Maduka IC: Atherogenic index of plasma as useful predictor of cardiovascular risk among postmenopausal women in Enugu, Nigeria. Afr Health Sci. 2010, 10:248-252.

11. Bora K, Pathak MS, Borah P, Hussain MI, Das D: Association of the apolipoprotein A-I gene polymorphisms with cardiovascular disease risk factors and atherogenic indices in patients from Assam, Northeast India. Balkan J Med Genet. 2017, 20:59-70. 10.1515/bjmg-2017-0002

12. Thiruvoipati T, Kielhorn CE, Armstrong EJ: Peripheral artery disease in patients with diabetes: epidemiology, mechanisms, and outcomes. World J Diabetes. 2015, 6:961-969. 10.4239/wjd.v6.i7.961

13. Grenon SM, Vittinghoff E, Owens CD, Conte MS, Whooley M, Cohen BE: Peripheral artery disease and risk of cardiovascular events in patients with coronary artery disease: insights from the Heart and Soul Study. Vasc Med. 2013, 18:176-184. 10.1177/1358863X13493825

14. Hinchliffe RJ, Brownrigg JR, Apelqvist J, et al.: IWGDF guidance on the diagnosis, prognosis and management of peripheral artery disease in patients with foot ulcers in diabetes. Diabetes Metab Res Rev. 2016, 32:37-44. 10.1002/dmrr.2698

15. Mehraj M, Shah I: A review of Wagner classification and current concepts in management of diabetic foot . Int J Orthop Sci. 2018, 41:933-935. 10.22271/ortho.2018.v4.i1n.133

16. Al-Rubeaan K, Al Derwish M, Ouizi S, Youssef AM, Subhani SN, Ibrahim HM, Alamri BN: Diabetic foot complications and their risk factors from a large retrospective cohort study. PLoS One. 2015, 10:124446. 10.1371/journal.pone.0124446

17. Bruun C, Siersma V, Guassora AD, Holstein P, de Fine Olivarius N: Amputations and foot ulcers in patients newly diagnosed with type 2 diabetes mellitus and observed for 19 years. The role of age, gender and comorbidity. Diabet Med. 2013, 30:964-972. 10.1111/dme.12196

18. Nehring P, Mrozikiewicz-Rakowska B, Krzyżewska M, Sobczyk-Kopcioł A, Płoski R, Broda G, Karnafel W: Diabetic foot risk factors in type 2 diabetes patients: a cross-sectional case control study . J Diabetes Metab Disord. 2014, 13:79. 10.1186/2251-6581-13-79

19. Al-Maskari F, El-Sadig M: Prevalence of risk factors for diabetic foot complications . BMC Fam Pract. 2007, 8:59. 10.1186/1471-2296-8-59

20. Al-Delaimy WK, Merchant AT, Rimm EB, Willett WC, Stampfer MJ, Hu FB: Effect of type 2 diabetes and its duration on the risk of peripheral arterial disease among men. Am J Med. 2004, 116:236-240. 10.1016/j.amjmed.2003.09.038

21. Hurley L, Kelly L, Garrow AP, et al.: A prospective study of risk factors for foot ulceration: the West of Ireland Diabetes Foot Study. QJM. 2013, 106:1103-1110. 10.1093/qjmed/hct182

22. Frykberg RG, Zgonis T, Armstrong DG, et al.: Diabetic foot disorders. A clinical practice guideline (2006 revision). J Foot Ankle Surg. 2006, 45:1-66. 10.1016/\$1067-2516(07)60001-5

23. Khan Y, Khan MM, Jain A, Namdev RK: A study of association of diabetic foot ulcers and peripheral vascular disease. Int J Adv Med. 2018, 5:1454-1459. 10.18203/2349-3933.ijam20184756

24. McIntyre KE: Control of infection in the diabetic foot: the role of microbiology, immunopathology, 


\section{Cureus}

antibiotics, and guillotine amputation. J Vasc Surg. 1987, 5:787-790. 10.1067/mva.1987.avs0050787b

25. Adler AI, Erqou S, Lima TA, Robinson AH: Association between glycated haemoglobin and the risk of lower extremity amputation in patients with diabetes mellitus-review and meta-analysis. Diabetologia. 2010, 53:840-849. 10.1007/s00125-009-1638-7

26. Selim S: Frequency and pattern of chronic complications of diabetes and their association with glycemic control. Diabetes Metab Syndr. 2017, 11:311-314. 10.1016/j.dsx.2017.03.007

27. Muhtaroğlu S, Barlak Keti D, Ünlühizarci K: Investigation of ischemia-modified albumin levels and some atherosclerosis-related serum parameters in patients with diabetic foot. Turk J Med Sci. 2016, 46:126-132. 10.3906/sag-1406-38

28. Nie X, Gao L, Wang L, Wang J: Atherogenic index of plasma: a potential biomarker for clinical diagnosis of diabetic foot osteomyelitis . Surg Infect (Larchmt). 2020, 21:9-14. 10.1089/sur.2019.020

29. Janka HU, Standl E, Mehnert H: Peripheral vascular disease in diabetes mellitus and its relation to cardiovascular risk factors: screening with the doppler ultrasonic technique. Diabetes Care. 1980, 3:207-213. 10.2337/diacare.3.2.207

30. Wen J, Zhong Y, Kuang C, Liao J, Chen Z, Yang Q: Lipoprotein ratios are better than conventional lipid parameters in predicting arterial stiffness in young men. J Clin Hypertens (Greenwich). 2017, 19:771-776. 10.1111/jch. 13038 